\title{
Le Lymphangiome kystique scrotal. A propos d'un cas
}

\author{
Hammadi FAKHFAKH, Abdelkader BOUHLEL, Hafedh KETATA, Ahmed SAHNOUN, \\ Ali BAHLOUL, Mohamed Nabil MHIRI
}

\author{
Service d'urologie du CHU Habib Bourguiba, Sfax - Tunisie
}

\section{RESUME}

Le lymphangiome est une tumeur bénigne rare du système lymphatique.

Il se voit surtout chez l'enfant, la localisation cervicale ou axillaire est la plus habituelle, celle du scrotum reste rare.

Nous rapportons l'observation d'un homme âgé de 39 ans qui avait consulté pour une masse indolore du scrotum gauche. L'examen avait révélé une masse indépendante du testicule. L'échographie était en faveur d'une masse kystique du scrotum. L'examen anatomo-pathologique avait conclu à un lymphangiome scrotal de type kystique. La masse avait été excisée complètement. Aucune récidive n'avait été observée après un suivi de 6 mois.

Mots clés : Iymphangiome, kyste, scrotum, chirurgie

\section{INTRODUCTION}

Le lymphangiome est une tumeur bénigne rare du système lymphatique [1].

Ces tumeurs sont observées habituellement chez les enfants et exceptionnellement chez l'adulte. Elles surviennent le plus fréquemment au niveau du cou (75\%) et de la région axillaire $(20 \%)$ [2]. La localisation scrotale demeure rare [3]. Le diagnostic en préopératoire d'un lymphangiome scrotal n'est pas toujours facile à faire.

\section{OBSERVATION}

Monsieur S.B. âgé de 49 ans, s'était présenté pour une masse indolore du scrotum gauche, évoluant depuis 3 semaines de façon progressive et responsable d'une gène fonctionnelle à type de pesanteur.
A l'interrogatoire, il n'y avait pas d'antécédent notable, en particulier pas de notion de traumatisme des bourses. L'examen révélait une masse de la partie inférieure du scrotum gauche de $10 \mathrm{~cm}$ de grand axe sans signes inflammatoires cutanés en regard. Cette masse était rénitente, en grappe de raisin, adhérente par endroit à la peau scrotale indépendante du testicule ainsi que du cordon (Figure 1). La transillumination était négative. Le testicule et le cordon contro-latéraux étaient normaux. Le reste de l'examen était normal.

L'échographie scrotale, couplée au doppler, avait montré une formation scrotale gauche de $9 \mathrm{~cm} / 5 \mathrm{~cm}$, composée de multiples formations liquidiennes séparées par des cloisons vascularisées et contenant des sédiments avec des niveaux liquide-liquide (Figure 2).

Les marqueurs tumoraux (l'alpha-foetoprotéine et la sous unité $ß$ de l'hormone chorionique gonadotrope) étaient normaux.

En préopératoire une tumeur para testiculaire multi kystique ou une hématocèle cloisonné furent évoquées, d'où une décision d'exploration chirurgicale par voie scrotale.

II s'agissait en fait d'une masse de $9 \mathrm{~cm} / 6,5 \mathrm{~cm}$, composée de multiples kystes de taille variable et remplis de sang noirâtre et non cailloté (Figure 3). Celle-ci était facilement clivable du testicule gauche mais adhérente par endroits à la paroi scrotale. Il existait une extension périnéale en profondeur arrivant jusqu'à l'urètre bulbaire sans l'envahir. Une sonde de type Foley à été mise en place en trans-urètral afin d'identifier l'urètre et faciliter sa dissection. La masse était excisée complètement en bloc emportant une partie de la peau scrotale adhérente.

\section{Correspondance :}

Dr Hammadi FAKHFAKH - service d'urologie, de CHU Habib Bourguiba, Sfax, Tunisie - Tel 0021698639784 Email h.fakhfakh@laposte.net 


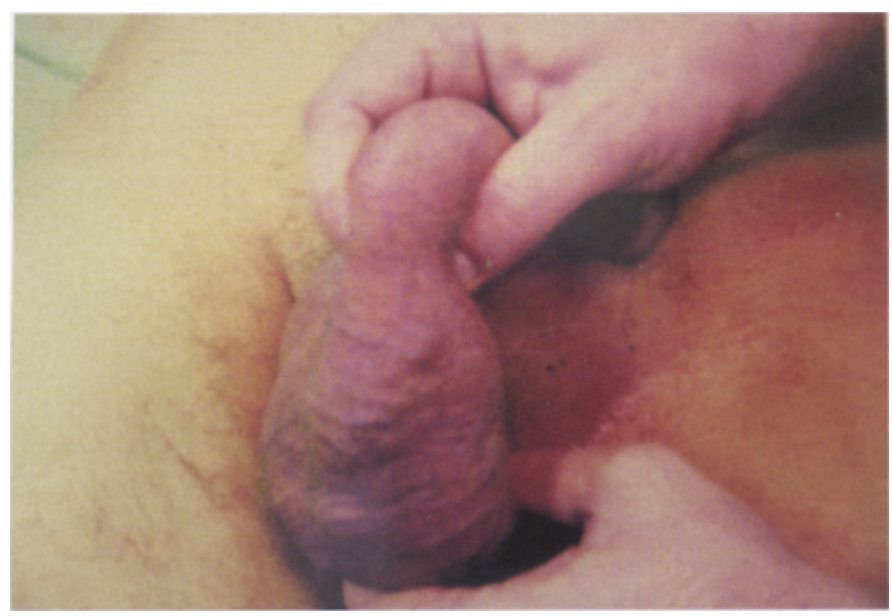

Figure 1 : masse scrotale indépendante du testicule gauche.

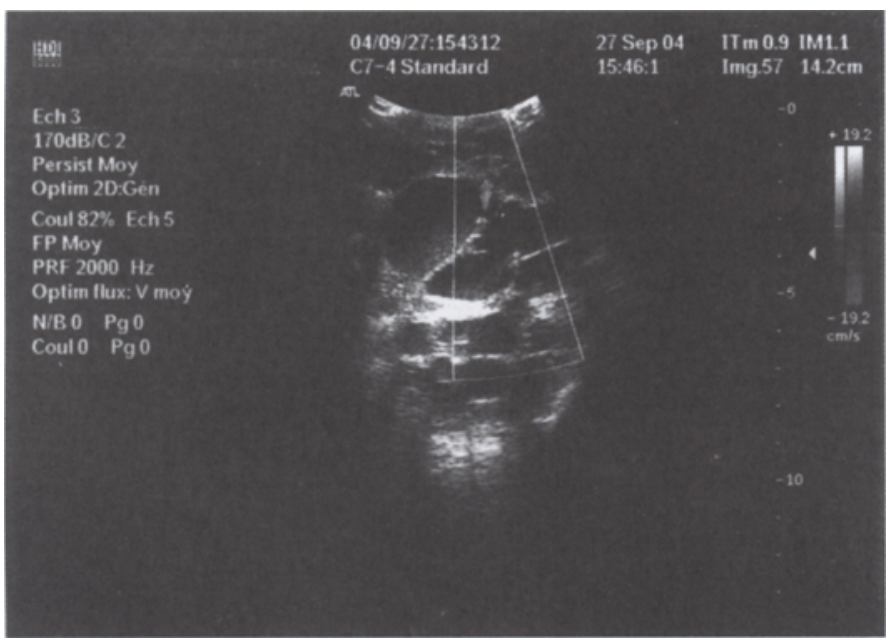

Figure 2 : aspect multikystique à l'échographie.

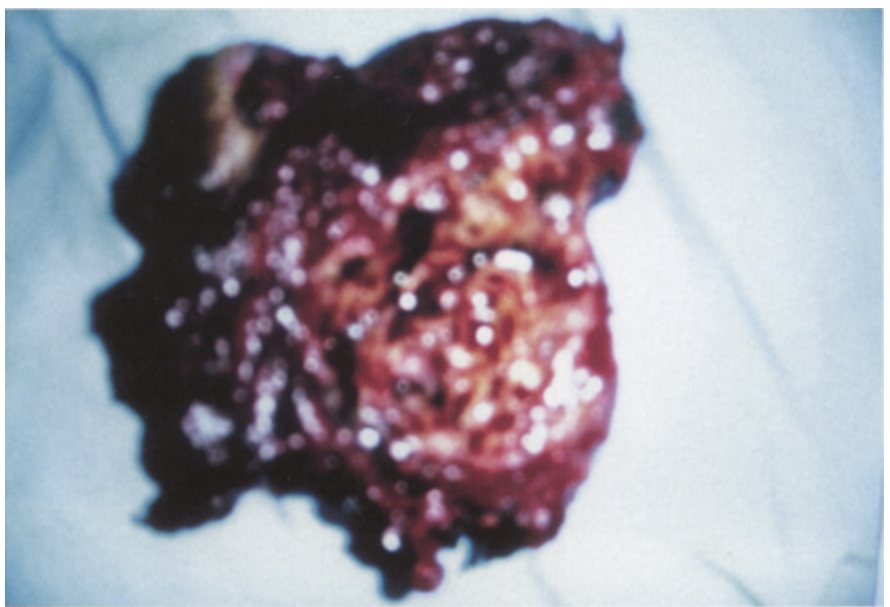

Figure 3 : exérèse complète avec la peau scrotale.
A l'examen anatomopathologique il s'agissait d'un lymphangiome scrotal de type kystique formé de dilatations kystiques des vaisseaux lymphatiques avec un contenu chyleux et hémorragique, renfermant dans leurs parois de petits amas lymphoïdes. II n'existait pas de signes de malignité.

Les suites opératoires immédiates et tardives étaient simples sans récidives avec un suivi de 6 mois.

\section{DISCUSSION}

Les lymphangiomes sont des tumeurs bénignes, qui ne dégénèrent jamais, classées selon l'importance de la dilatation des espaces lymphatiques en trois types : simple (capillaire), caverneux et kystique [5]. Ces tumeurs atteignent le plus souvent les enfants. Près de la moitié des lymphangiomes sont découverts à la naissance. II sont diagnostiqués avant l'âge de 2 ans dans $90 \%$ des cas [2].

Ce sont des malformations congénitales du système lymphatique. Cependant certains auteurs suggèrent une origine acquise résultant d'une obstruction des vaisseaux lymphatiques à la suite d'une inflammation ou d'un traumatisme [1 - 3]. Ces tumeurs surviennent le plus fréquemment au niveau du cou $(75 \%)$ et de la région axillaire (20\%) [2]. La localisation scrotale est rare, 45 cas auraient été rapportés dans la littérature [1].

Habituellement, les lymphangiomes scrotaux (LS) se présentent comme une masse scrotale indolore d'évolution progressive, gênant le patient physiquement et esthétiquement $[2,3]$. A l'inspection, cette masse parait lobulée. La peau en regard peut être bleutée ou violacée, cela étant dû au contenu sanguin des espaces kystiques. Rarement, la peau parait être érythémateuse, en cas d'infection associée [1 - 4]. Cette masse peut être molle, ferme et/ou tendue. Elle est le plus souvent solidaire de la peau scrotale. Le testicule et le cordon sont palpables et facilement dissociables de la lésion scrotale [1 - 3].

L'échographie permet le plus souvent de mettre en évidence une masse scrotale multikystique non vascularisée. Certains kystes peuvent contenir du matériel finement échogène correspondant à du sang [1 - 3]. Mais, contrairement à ce qui a été rapporté dans la littérature, dans notre observation, une vascularisation des parois kystiques avait été notée.

Par ailleurs, l'imagerie par résonance magnétique (IRM) permettrait d'évoquer le diagnostic et d'éliminer une hydrocèle cloisonnée et une spermatocèle. Typiquement, il s'agit d'une lésion hétérogène en hypo signal en T1 et en hyper signal en T2, reflétant le contenu lymphatique des cavités kystiques. En cas d'hémorragie intra-kystique, les kystes apparaissent en hypo signal en T2. L'IRM permet d'apprécier l'extension de la lésion par rapport aux tissus mous adjacents et aux structures vasculaires [1, 7]. Enfin, La ponction des kystes avec analyse de leur contenu liquidien montrant la présence de liquide lymphatique pourrait orienter le diagnostic [2].

La chirurgie reste le traitement de référence des lymphan- 
giomes scrotaux $[1-3,5]$. La voie d'abord est scrotale. L'excision complète de la tumeur permettrait de prévenir la récidive [1, 2]. Habituellement, les lymphangiomes scrotaux sont plus étendus que prévu et peuvent avoir une extension pelvienne, rétro péritonéale ou périnéale profonde amenant ainsi à une dissection extensive afin d'enlever toute la tumeur [3].

Le testicule et le cordon sont normaux et dissociables du lymphangiome [3]. La peau scrotale recouvrant le lymphangiome est le plus souvent excisée avec la masse [3,5]. Comme cela en est le cas dans notre observation, et dans ce cadre, en présence d'un plan de clivage avec la peau, l'excision cutanée n'est pas obligatoire [1,2].

Pour les lymphangiomes volumineux inextirpables, certains auteurs proposent une sclérothérapie par injection intrakystique de OK-432 [6]. C'est ainsi que Uchida et al. avaient traité avec succès un énorme lymphangiome scrotal avec extension rétro-péritonéale [8].

\section{CONCLUSION}

Le lymphangiome kystique scrotal est une tumeur bénigne rare. La symptomatologie clinique est non spécifique. Le diagnostic, suspecté par l'imagerie devant une masse kystique, doit être confirmé histologiquement.

Le traitement de choix est chirurgical consistant en une exérèse complète de la lésion. Son pronostic est bénin avec seulement un risque de récidive en cas de résection incomplète.

\section{REFERENCES}

1. HAMADA Y., YAGI K., TANANO A. et al. : Cystic lymphangioma of the scrotum. Pediatr. Surg. Int., 1998, $13: 442-444$.

2. HURWITZ R.S., SHAPIRO E., HULBERT W.C., DIAMOND D.A., CASALE A.J., RINK R.C: J. : Scrotal cystic lymphangioma : the misdiagnosed scrotal mass. Urol., 1997, 158 : 11821185.

3. KINGSLY K.A., SAINT G.R. : Scrotal lymphangioma in adult. Urol., 1999, $53: 820-821$.

4. MERKA S.T., BHATT K.S., WOOD F.W. : Cystic lymphangioma of scrotum. J. Urol., 1984, $131:$ 1179-1180.

5. MULCAHY J.J., SCHILERU G., DONEMEZER M.A., BHATHEMA D. : Lymphangioma of scrotum. Urology, 1979, 14 : 64-65.

6. OGITA S., TSUTO T., DEGUCHI E. : OK-432 therapy for unresectable lymphangiomas in children. J. Pediatr. Surg., 1991, 26: 263-270.

7. SIEGEL M.J., GLAZER H.S., ST-AMOUR T.E., ROTHENTHAL D.D. : Lymphangiomas in children : MR imaging. Pediatr. Radiol., 1989, $170: 467-470$

8. UCHIDA K., INOUE M., ARAKI T., MIKI C., KUSUNOKI M. : Huge scrotal, flank, and retroperitoneal lymphangioma successfully treated by OK-432 sclerotherapy. Urol., 2002, 60 :2426.

Manuscrit reçu : mars 2005 ; accepté avril 2005.

\begin{abstract}
Scrotal cystic lymphangioma : a case report.

Hammadi FAKHFAKH, Abdelkader BOUHLEL, Hafedh KETATA, Ahmed SAHNOUN, Ali BAHLOUL, Mohamed Nabil MHIRI
\end{abstract}

Lymphangioma is a benign tumor of the lymphatic system classified as simple, cavernous, or cystic depending on the size of the dilated lymphatic spaces within the tumor. Most cases present as a soft, cystic, slowly enlarging swelling in the neck and axilla in children, while the scrotum is a rare site.

We report the case of a 39-year-old man who presented with a painless left hemiscrotal mass. On examination, the testes and spermatic cords were normal bilaterally. There was a firm, polycystic mass in the inferior portion of the left hemiscrotum.

Scrotal ultrasound revealed normal testes, with a large complex cystic loculated mass. Histology demonstrated a cystic lymphangioma. The mass was completely excised. The patient remained well without recurrence with a follow-up of 6 months.

Key-words : lymphangioma, cystic, scrotum, surgery 